\title{
The Vascular-Renal Connection in Patients Hospitalized With Hypertensive Crisis: A Population-Based Study
}

\author{
Siu-Hin Wan, MD; Joshua P. Slusser, BS; David O. Hodge, MS; \\ and Horng $H$. Chen, MBBCh
}

\begin{abstract}
Objective: To determine the risks of acute kidney injury development and long-term clinical outcomes of patients with hypertensive crisis.

Patients and Methods: This was a population study of Olmsted County residents with hypertensive crisis between January 1, 2000, and December 31, 2008, with follow-up until June 30, 2016.

Results: The results demonstrated that those with underlying chronic kidney disease upon admission for hypertensive crisis, defined as a systolic blood pressure above $180 \mathrm{~mm} \mathrm{Hg}$ or diastolic blood pressure above $120 \mathrm{~mm} \mathrm{Hg}$, were more likely to develop acute kidney injury during hospitalization (odds ratio, $6.04 ; 95 \% \mathrm{CI}, 1-26 ; P=.02$ ). Hospitalization length of stay was increased when patients developed acute kidney injury during hypertensive crisis hospitalization ( $7.6 \pm 9$ vs $3.4 \pm 4$ days; $P=.04$ ). Furthermore, those who developed acute kidney injury had increased cardiac rehospitalization frequency over 10 years ( $87 \%$ vs $46 \% ; P=.009$ ). These results suggest that those with poor renal reserve are more likely to have further acute kidney damage in the setting of hypertensive crisis, likely due to decreased renal perfusion and neurohormonal dysregulation.

Conclusion: In patients hospitalized for hypertensive crisis, chronic renal insufficiency was a risk factor associated with acute kidney injury development during hospitalization. Those who developed acute kidney injury had longer hospitalizations with increased rehospitalization frequency. Future studies are warranted to further investigate whether the preservation of renal function will improve clinical outcomes in hospitalized patients with hypertensive crisis.

(C) 2018 THE AUTHORS. Published by Elsevier Inc on behalf of Mayo Foundation for Medical Education and Research. This is an open access article under the CC BY-NC-ND license (http://creativecommons.org/licenses/by-nc-nd/4.0/) — Mayo Clin Proc Inn Qual Out 2018;2(2):148-154
\end{abstract}

From the Department of Cardiovascular Diseases (S.H.W., H.H.C.) and Division of Biomedical Statistics and Informatics (J.P.S., D.O.H.) Mayo Clinic, Rochester, MN

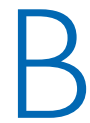
oth renal insufficiency and hypertension are extremely prevalent in the general population, and the occurrences of these conditions are intertwined. As many as $33 \%$ of adults in the United States have high blood pressure, and the prevalence of chronic kidney disease (CKD) in the United States is more than $16 \%{ }^{1}$ Hypertension hospitalizations are also a large burden to the US health care system, with 579,000 patients in 2009 discharged from a hospital in the United States for hypertension as the primary diagnosis. ${ }^{1}$

While it has been well documented that chronic hypertension negatively impacts kidney function, the long-term outcomes of patients hospitalized for hypertensive crisis with acute kidney injury (AKI) remain undefined. Previous studies have shown that in the short term, hospitalized patients with CKD who develop AKI have associated increased mortality. ${ }^{2-4}$ In the Studying the Treatment of Acute Hypertension registry, hospitalized acute severe hypertension patients with concurrent CKD and subsequent AKI had an increased risk of short-term mortality and morbidity. ${ }^{5}$ However, the longterm outcomes following renal dysfunction after an acute episode of severe hypertension remain undefined.

This study aimed to identify the risk factors associated with AKI development in the hypertensive crisis population and how an episode of AKI in this population affects long-term mortality, morbidity, and rehospitalization. Clarifying this vascular-renal relationship can help the clinician provide a 
more accurate prognosis to patients as well as become more alert to patients at high risk for end-organ dysfunction, rehospitalization, or death.

\section{PATIENTS AND METHODS}

\section{Study Setting}

The study protocol was approved by the institutional review boards of both Mayo Clinic and Olmsted Medical Center, and data were obtained from the Rochester Epidemiology Project, which maintains a medical record database for residents of Olmsted County, Minnesota. ${ }^{6}$ The Rochester Epidemiology Project database contains documentation of outpatient visits, emergency department visits, and hospitalizations.

In this retrospective analysis, we collected demographic, clinical, and laboratory data on adult residents of Olmsted County, Minnesota, who were hospitalized for hypertensive crisis between January 1, 2000, and December 31, 2008, with follow-up until June 30, 2016. Inclusion criteria were male and female adults, 18 years or older, who were hospitalized with a main discharge diagnosis of hypertensive crisis, defined as systolic blood pressure above $180 \mathrm{~mm} \mathrm{Hg}$ or diastolic blood pressure above $120 \mathrm{~mm} \mathrm{Hg}$, and identified with International Classification of Diseases, Ninth Revision codes and confirmed with detailed review of the medical records. Selected 401-405 International Classification of Diseases, Ninth Revision codes corresponding to hypertensive crisis were used. Only those patients with repeated creatinine measurements during hospitalization were included.

\section{Data Collection}

Collection of baseline characteristics for the identified patients included age, sex, ethnicity, body mass index (calculated as the weight in kilograms divided by the height in meters squared), history of hypertension, tobacco use, known coronary artery disease, and history of heart failure (HF). Collection of admission laboratory values included lipid panel, liver function tests, serum urea nitrogen (BUN), and plasma creatinine. Vitals signs, including admission blood pressure and highest blood pressure during hospitalization, were collected. The highest BUN and plasma creatinine values during hospitalization were also collected. Outcome variables collected include mortality and date of death, length of hospitalization, date and reason of first rehospitalization, and posthospitalization BUN and creatinine. Mortality data were collected from the Mayo Clinic or Olmsted Medical Center electronic medical records. Data on echocardiographic parameters within 6 months of hospitalization and admission and discharge blood pressure medications were collected.

Acute kidney injury development was defined as an increase of $0.3 \mathrm{mg} / \mathrm{dL}$ or $25 \%$ in plasma creatinine during the hospitalization. Glomerular filtration rate (GFR) was calculated by the Modification of Diet in Renal Disease method: estimated GFR (eGFR; mL/ $\left.\mathrm{min} / 1.73 \mathrm{~m}^{2}\right)=175 \times(\mathrm{Scr})^{-1.154} \times$ $(\text { Age })^{-0.203} \times(0.742$ if female $) \times(1.212$ if African American). Chronic kidney disease was defined by eGFR of less than $60 \mathrm{~mL} / \mathrm{min}$. Cardiac rehospitalization was defined as a rehospitalization with a main discharge diagnosis related to hypertension, coronary artery disease, arrhythmia, or HF.

\section{Data Analysis and Outcomes}

The primary analysis involved identifying risk factors for AKI development and determining the relationship between CKD and AKI among the hypertensive crisis population. Additional analyses included short- and long-term rehos pitalization and mortality outcomes for the hypertensive crisis population with or without AKI development during hospitalization.

Continuous variables are presented as mean $\pm S D$. Discrete variables are summarized as frequency (percentage). Comparisons between groups were made using the 2sample $t$ test for continuous variables and the Pearson $\chi^{2}$ test for categorical variables. Further assessment of potential associations with renal failure was done using a logistic regression model, adjusting for age and sex on selected variables that showed statistical significance. Long-term follow-up event estimates were computed using the KaplanMeier method, with group comparisons made via the log-rank test. Modeling of longterm outcomes was assessed using Cox proportional hazards models, again adjusting for age and sex on selected variables that showed 


\begin{tabular}{|c|c|c|c|}
\hline Variable & Overall $(\mathrm{N}=50)$ & AKI $(n=23)$ & No AKI $(n=27)$ \\
\hline Age $(y)$, mean \pm SD & $66.9 \pm 18.0$ & $64.3 \pm 18.3$ & $69.0 \pm 17.9$ \\
\hline \multicolumn{4}{|l|}{ Sex, No. (\%) } \\
\hline Male & $21(42)$ & $9(39)$ & $12(44)$ \\
\hline Female & $29(58)$ & $14(61)$ & $15(56)$ \\
\hline \multicolumn{4}{|l|}{ Ethnicity, No. (\%) } \\
\hline Black & I (2) & $0(0)$ & । (4) \\
\hline Asian & $3(6)$ & $2(9)$ & I (4) \\
\hline White & $46(92)$ & $21(91)$ & $25(93)$ \\
\hline Body mass index $\left(\mathrm{kg} / \mathrm{m}^{2}\right)$, mean $\pm \mathrm{SD}$ & $27.0 \pm 6.2$ & $27.6 \pm 8.2$ & $26.5 \pm 3.7$ \\
\hline \multicolumn{4}{|l|}{ Comorbidities, No. (\%) } \\
\hline History of smoking & $29(60)$ & II (48) & $18(72)$ \\
\hline History of hypertension & $43(86)$ & $20(87)$ & $23(85)$ \\
\hline History of CAD & $13(26)$ & $6(26)$ & $7(26)$ \\
\hline History of MI & $6(12)$ & $2(9)$ & $4(15)$ \\
\hline History of DM & $12(24)$ & $6(26)$ & $6(22)$ \\
\hline History of heart failure & $5(10)$ & $2(9)$ & $3(11)$ \\
\hline Total cholesterol (mg/dL), mean $\pm \mathrm{SD}$ & $182.2 \pm 40.0$ & $183.3 \pm 44.2$ & $181.2 \pm 36.5$ \\
\hline Ejection fraction (\%) & $56.9 \pm 12.0$ & $56.5 \pm 13.9$ & $57.4 \pm 9.9$ \\
\hline GFR $\left(\mathrm{mL} / \mathrm{min} / 1.73 \mathrm{~m}^{2}\right)$, mean $\pm \mathrm{SD}$ & $50.9 \pm 24.8$ & $41.3 \pm 26.5$ & $59.1 \pm 20.4$ \\
\hline GFR $<60 \mathrm{~mL} / \mathrm{min} / \mathrm{l} .73 \mathrm{~m}^{2}$, No. (\%) & $34(68)$ & $20(87)$ & $14(52)$ \\
\hline Highest systolic BP (mm Hg), mean \pm SD & $230.7 \pm 21.8$ & $236.6 \pm 23.8$ & $225.6 \pm 18.9$ \\
\hline Highest diastolic BP ( $\mathrm{mm} \mathrm{Hg})$, mean $\pm \mathrm{SD}$ & $110.8 \pm 24.8$ & || $1.0 \pm 25 . \mid$ & । $10.7 \pm 25.0$ \\
\hline No. of comorbidities, mean \pm SD & $2.3 \pm 1.2$ & $2.3 \pm 1.5$ & $2.3 \pm 1.0$ \\
\hline No. of discharge medications, mean $\pm \mathrm{SD}$ & $2.9 \pm 1.6$ & $3.0 \pm 1.8$ & $2.9 \pm 1.4$ \\
\hline Length of hospitalization stay (d), mean \pm SD & $5.3 \pm 7.1$ & $7.6 \pm 9.2$ & $3.4 \pm 3.8$ \\
\hline
\end{tabular}

statistical significance. Statistical significance is defined as a 2-tailed $P$ value of less than .05 . Statistical analyses were completed with SAS 9.4 (SAS Institute).

\section{RESULTS}

\section{Baseline Characteristics}

Between January 1, 2000, and December 31, 2008, there were 50 patients from Olmsted County who met the inclusion criteria of having a primary hospitalization discharge diagnosis of hypertensive crisis (defined as systolic blood pressure above $180 \mathrm{~mm} \mathrm{Hg}$ or diastolic blood pressure above $120 \mathrm{~mm} \mathrm{Hg}$ ), with creatinine monitoring during hospitalization. Demographic analysis showed the mean age of the study population to be 67 years, with a larger proportion being female (58\%). A large proportion of patients, $68 \%$, had CKD as defined by an eGFR of less than $60 \mathrm{~mL} / \mathrm{min}$ calculated by the Modification of Diet in Renal Disease method. The most prevalent cardiovascular comorbidity was hypertension (86\%). Table 1 presents the full baseline characteristics, including demographic data and cardiovascular comorbidities, of the overall study population subdivided into those who did or did not develop AKI during hospitalization.

\section{Risk of AKI Development}

The 50 patients identified for this study were subdivided into those who, during hospitalization, did or did not develop AKI. Those in the AKI group had lower average admission GFR compared with those in the non-AKI group (41.3 vs $59.1 \mathrm{~mL} / \mathrm{min}$ per $1.73 \mathrm{~m}^{2} ; P=.01$ ). There was a greater proportion of those with reduced GFR on admission in the AKI vs non-AKI group ( $87 \%$ vs $52 \%$; $P=.008$ ). Multivariate analyses with age and sex adjustment showed that those with underlying CKD were more likely to develop AKI during hospitalization (odds ratio, 6.04; 95\% CI, 1-26; $P=.02)$. 


\begin{tabular}{|c|c|c|c|c|}
\hline Variable & Overall $(\mathrm{N}=50)$ & AKI $(n=23)$ & No AKI $(n=27)$ & $P$ value \\
\hline ACE inhibitors & I (2) & I (4) & $0(0)$ & .27 \\
\hline Calcium channel blockers & $3(6)$ & $2(9)$ & I (4) & .46 \\
\hline Nitrates & $12(24)$ & $7(30)$ & $5(19)$ & .33 \\
\hline Diuretics & $5(10)$ & $4(17)$ & I (4) & .11 \\
\hline$\beta$-Blockers & $18(36)$ & $9(39)$ & $9(33)$ & .67 \\
\hline Benzodiazepine & I (2) & I (4) & $0(0)$ & .27 \\
\hline Hydralazine & $2(4)$ & $0(0)$ & $2(7)$ & .18 \\
\hline
\end{tabular}

The in-hospital types of antihypertensive medications administered among those who did develop AKI vs those who did not are presented in Table 2.

\section{Hospitalization Duration}

Among those hospitalized for hypertensive crises, those who developed AKI irrespective of the baseline eGFR had a significantly longer hospital length of stay (7.6 \pm 9 vs $3.4 \pm 4$ days; $P=.04$ ). Furthermore, multivariate analysis with age and sex adjustment revealed that AKI development during hypertensive crisis hospitalization was associated with longer length of hospitalization (parameter estimate, 4.64; 95\% CI, 0.78-8.50; $P=.02$ ).

\section{Long-term Outcomes for Those Hospitalized With Hypertensive Crisis}

Cardiac Rehospitalization. Those who developed AKI had increased frequency of cardiac rehospitalization, both at 3 years $(62 \%$ vs $24 \% ; P=.03$ ) and at 10 years ( $87 \%$ vs $46 \%$; $P=.009$ ) (Figure 1). Multivariate analysis with age and sex adjustment showed that AKI development was a predictor of cardiac rehospitalization (hazard ratio, 4.20; 95\% CI, 1.859.52; $P<.001)$. Use of a particular antihypertensive medication class on admission, such as angiotensin-converting enzyme inhibitors, $\beta$-blockers, diuretics, calcium channel blockers, or nitrates, was not found to be associated with increased cardiac rehospitalization rates.

Heart Failure. There was no significant association between AKI development and rehospitalization due to HF $(P=.09)$, or incident HF $(P=.56)$.
Mortality. Among those hospitalized with hypertensive crisis, there was no statistically significant difference in 1-, 3-, or 10-year mortality among groups with or without CKD $(P>.05)$. Overall, there was no significant association between AKI development and mortality $(P=.78)$ (Figure 2).

Multivariate analysis, adjusting for age and sex, did not show that length of hospitalization (hazard ratio, 1.04; 95\% CI, 0.99-1.09; $P=.09$ ) was a predictor of all-cause mortality among those hospitalized with hypertensive crisis.

\section{DISCUSSION}

The main findings of this study are as follows: underlying CKD was a risk factor for AKI development during hospitalization for a hypertensive crisis; and those who developed AKI during hospitalization had worse shortand long-term rehospitalization outcomes.

The results of this study demonstrated that those with underlying CKD are predisposed to AKI development in hypertensive crisis. While the exact pathophysiologic mechanism remains incompletely defined, it can be hypothesized that those with poor renal reserve, coupled with a hypertensive crisis, are more likely to develop decreased renal perfusion and neurohormonal dysregulation. Chronic hypertension detrimentally affects kidney function, and hypertension leading to end-stage renal disease has been extensively studied. The pathophysiology includes renal vasculature remodeling and dysregulation of the renin-angiotensin-aldosterone pathway. Poor renal function can lead to alterations in blood pressure by fluid imbalance and dysfunction of the renin-angiotensin-aldosterone system. 


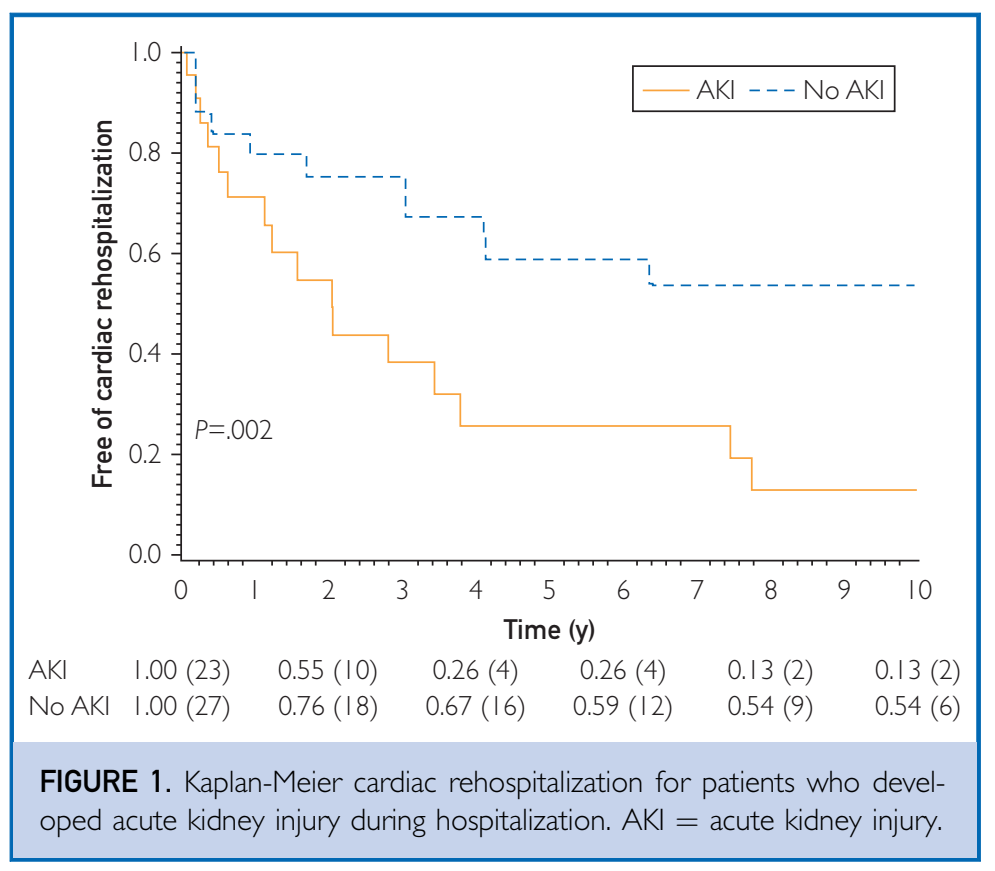

Furthermore, evaluation of hypertensive crisis patients revealed that plasma renin and aldosterone were markedly elevated, suggesting neurohormonally mediated renal dysfunction and vascular damage. ${ }^{8}$

In addition to neurohormonal dysfunction in hypertensive crisis, kidney injury results

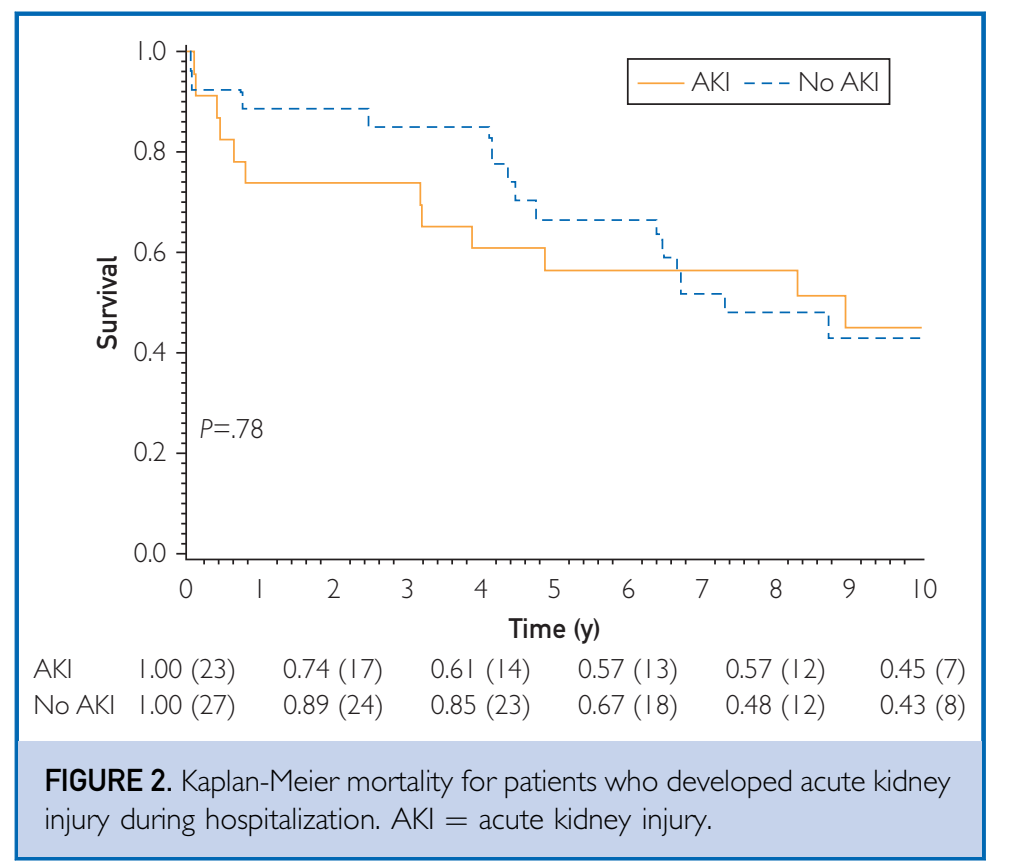

from abnormalities in renal vascular autoregulation. ${ }^{9}$ Normally, when renal perfusion pressure decreases and blood flow to the kidneys is reduced, autoregulation results in the reduction of vascular resistance to normalize blood flow. However, in hypertensive crisis, the kidney's autoregulatory mechanism is impaired. Additional mechanisms for vascular injury and renal dysfunction include immuneand inflammatory-mediated fibrosis. Histological evaluation of patients with hypertensive crisis associated with nephropathy in previous studies has demonstrated an arteriolar onion skin pattern on renal biopsy. ${ }^{10}$ Animal models have been used to demonstrate the importance of inflammation and stress in hypertensivemediated vascular and renal injury. ${ }^{11}$ Methylglyoxal, part of the carbonyl stress pathway, has been implicated in hypertension-mediated renal injury and kidney sclerosis due to inflammation and oxidative stress. ${ }^{12}$ Similarly, chemokine ligand 16 may be responsible for immune-mediated renal dysfunction in hypertension, and knockout mice demonstrated protective features against renal fibrosis and dysfunction. ${ }^{13}$ In summary, dysfunction of the renin-angiotensin-aldosterone system, dysfunction of autoregulation, and inflammation- and immune-mediated renal vascular fibrosis may contribute to kidney injury with hypertensive crisis. The findings in this study are likely multifactorial, and further mechanistic research is necessary to elucidate the pathophysiology of AKI in hypertensive crisis.

The findings of this study are consistent with and extend previous research in hypertensive crisis. Szczech et $\mathrm{al}^{5}$ concluded, on the basis of Studying the Treatment of Acute Hypertension registry, that patients admitted to the hospital with acute severe hypertension often have concurrent $\mathrm{CKD}^{5}$ and that additional AKI leads to an increased risk of short-term (90-day) mortality and morbidity. ${ }^{5}$ The current study is the first, to our knowledge, to report increased long-term morbidity (up to 10 years) associated with AKI development in the hypertensive crisis population. Specifically, we found that increased cardiac rehospitalization rates persisted up to 10 years. Furthermore, although there was a trend for greater mortality up to 3 years among the AKI population, there was no mortality difference at 10 years. 


\section{Limitations}

This is a single-center retrospective analysis of patients hospitalized for hypertensive crisis. Given the nature of this study, no causal relationship can be inferred given the presence of possible confounders contributing to renal dysfunction. While one of the strengths of the study is the close follow-up of patients given that they were from a single county, the demographic characteristics may also limit generalizability or extrapolation of results to different populations. The use of coding data is a limitation of this study. Although the medical records of patients were reviewed to confirm that hypertensive crisis was present, there may have been selection bias because some patients with hypertensive crisis during the study period may not have been included if the appropriate diagnoses were not coded by the clinician. The study population is predominantly white, and thus this limits extrapolation of outcomes in other racial and ethnic groups. The retrospective nature of this study also limited the amount of renal injury markers that could be collected. Study limitations also include the small sample size, which may increase the risk for type I and type II errors. The study did not find any association between AKI and mortality, and this may be due to the low power of the study. Future prospective studies with longitudinal follow-up and frequent creatinine measures may investigate the long-term progression of CKD after hospitalization for hypertensive crisis and AKI.

\section{Perspectives}

This study demonstrated underlying CKD to be a risk factor for AKI development during hospitalization for a hypertensive crisis. Furthermore, those who developed AKI during hospitalization had worse short- and long-term rehospitalization outcomes. Dysfunction of the reninangiotensin-aldosterone system, dysfunction of autoregulation, and inflammation- and immune-mediated renal vascular fibrosis may be important in the pathophysiology of renal dysfunction with hypertensive crisis. Future large prospective studies are necessary to both identify those at risk of AKI development and tailor medication management and antihypertensive regimen to best preserve renal function and improve long-term outcomes. Currently, drug selection for hypertensive crisis management is highly provider-dependent, and therefore, a better understanding of the different phenotypes of the hospitalized population will help guide the clinician in choosing the most appropriate agent. Renal preservation should be a main goal of hypertensive crisis treatment, and the intricate cardiorenal relationship in acute hypertension may result in the development of a new generation of pharmacologic agents that have both beneficial blood pressure lowering and renal perfusion effects. ${ }^{14,15}$

\section{CONCLUSION}

In patients hospitalized for hypertensive crisis, chronic renal insufficiency was a risk factor associated with AKI development during hospitalization. Those who developed AKI had longer hospitalizations with increased rehospitalization frequency. Future studies are warranted to determine whether the preservation of renal function will improve outcomes in hospitalized patients with hypertensive crisis.

Abbreviations and Acronyms: AKI = acute kidney injury; BUN = blood urea nitrogen; $\mathbf{C K D}=$ chronic kidney disease; eGFR = estimated glomerular filtration rate; GFR = glomerular filtration rate; $\mathbf{H F}=$ heart failure

Potential Competing Interests: Dr Chen is cofounder of Zumbro Discovery. The rest of the authors report no competing interests.

Correspondence: Address to Horng $\mathrm{H}$. Chen, MBBCh, Mayo Clinic, 200 First St SW, Rochester, MN 55905 (chen.homg@mayo.edu).

\section{REFERENCES}

1. Writing Group Members, Mozaffarian D, Benjamin EJ, Go AS, et al; American Heart Association Statistics Committee; Stroke Statistics Subcommittee. Heart disease and stroke statistics - 2016 update: a report from the American Heart Association. Circulation. 2016;133(4):e38-e360.

2. Collins AJ, Li S, Gilbertson DT, Liu J, Chen SC, Herzog CA. Chronic kidney disease and cardiovascular disease in the Medicare population. Kidney Int Suppl. 2003:87:S24-S3I.

3. Chertow GM, Burdick E, Honour M, Bonventre JV, Bates DW. Acute kidney injury, mortality, length of stay, and costs in hospitalized patients. J Am Soc Nephrol. 2005; I 6(I I):3365-3370.

4. Go AS, Chertow GM, Fan D, McCulloch CE, Hsu CY. Chronic kidney disease and the risks of death, cardiovascular events, and hospitalization. N Engl J Med. 2004;35 I (I3): | 296- I 305.

5. Szczech LA, Granger CB, Dasta JF, et al; Studying the Treatment of Acute Hypertension Investigators. Acute kidney injury and cardiovascular outcomes in acute severe hypertension. Circulation. 2010; 121 (20):2183-2191

6. Melton LJ III. History of the Rochester Epidemiology Project. Mayo Clin Proc. 1996;7I (3):266-274. 
7. Collins AJ, Foley RN, Herzog C, et al. Excerpts from the US Renal Data System 2009 Annual Data Report. Am J Kidney Dis. 20 I0:55(I, suppl I):SI-S420. A426-A427.

8. van den Born BJ, Koopmans RP, van Montfrans GA. The reninangiotensin system in malignant hypertension revisited: plasma renin activity, microangiopathic hemolysis, and renal failure in malignant hypertension. Am J Hypertens. 2007;20(8):900-906.

9. Taylor DA. Hypertensive crisis: a review of pathophysiology and treatment. Crit Care Nurs Clin North Am. 2015;27(4):439-447.

10. Nonaka K, Ubara Y, Sumida $K$, et al. Clinical and pathological evaluation of hypertensive emergency-related nephropathy. Intern Med. 20 I3;52(I):45-53.

11. Schiffrin EL. Immune mechanisms in hypertension and vascular injury. Clin Sci (Lond). 20 I4; I 26(4):267-274.
12. Chen $\mathrm{X}$, Mori T, Guo Q, et al. Carbonyl stress induces hypertension and cardio-renal vascular injury in Dahl salt-sensitive rats. Hypertens Res. 2013;36(4):361-367.

13. Xia Y, Entman ML, Wang Y. Critical role of CXCLI 6 in hypertensive kidney injury and fibrosis. Hypertension. 2013;62(6): |129-1137.

14. McKie PM, Cataliotti A, Huntley BK, Martin FL, Olson TM, Burnett JC Jr. A human atrial natriuretic peptide gene mutation reveals a novel peptide with enhanced blood pressurelowering, renal-enhancing, and aldosterone-suppressing actions. J Am Coll Cardiol. 2009:54(I I): 1024- 1032.

15. McKie PM, Cataliotti A, Boemigter G, et al. A novel atrial natriuretic peptide based therapeutic in experimental angiotensin II mediated acute hypertension. Hypertension. 20 I 0;56(6): I I52-I I59. 